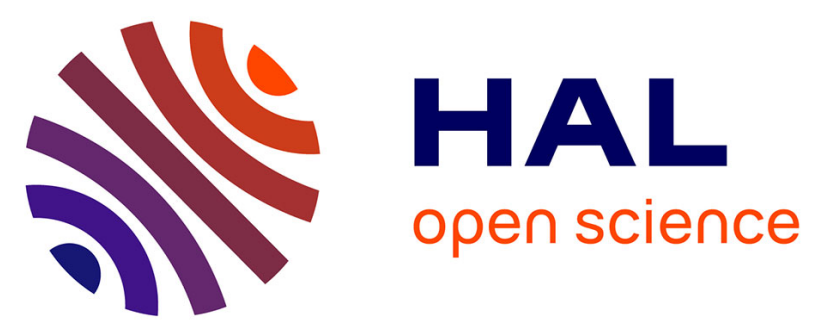

\title{
Toward full carbon interconnects: High conductivity of individual carbon nanotube to carbon nanotube regrowth junctions
}

Sampo Tuukkanen, Stephane Streiff, Pascale Chenevier, M. Pinault, Hee J. Jeong, Shaima Enouz-Vedrenne, Costel Sorin Cojocaru, Didier Pribat, Jean P. Bourgoin

\section{To cite this version:}

Sampo Tuukkanen, Stephane Streiff, Pascale Chenevier, M. Pinault, Hee J. Jeong, et al.. Toward full carbon interconnects: High conductivity of individual carbon nanotube to carbon nanotube regrowth junctions. Applied Physics Letters, 2009, 95, pp.113108. 10.1063/1.3216839 . hal-00794045

\section{HAL Id: hal-00794045 \\ https://hal.science/hal-00794045}

Submitted on 2 Mar 2013

HAL is a multi-disciplinary open access archive for the deposit and dissemination of scientific research documents, whether they are published or not. The documents may come from teaching and research institutions in France or abroad, or from public or private research centers.
L'archive ouverte pluridisciplinaire $\mathbf{H A L}$, est destinée au dépôt et à la diffusion de documents scientifiques de niveau recherche, publiés ou non, émanant des établissements d'enseignement et de recherche français ou étrangers, des laboratoires publics ou privés. 


\title{
Toward full carbon interconnects: High conductivity of individual carbon nanotube to carbon nanotube regrowth junctions
}

\author{
Pribat $^{3}$ and J.-P. Bourgoin ${ }^{1}$ \\ ${ }^{1}$ Laboratoire d'Electronique Moléculaire, IRAMIS/SPEC (CEA-CNRS URA 2464), \\ 91191 Gif-sur-Yvette, France \\ ${ }^{2}$ Laboratoire Francis Perrin (CEA-CNRS URA 2453), IRAMIS/SPAM, CEA/Saclay, \\ 91191 Gif-sur-Yvette, France \\ ${ }^{3}$ LPICM, Ecole Polytechnique-CNRS UMR 7647, 91128 Palaiseau, France
}

S. Tuukkanen, ${ }^{1}$ S. Streiff, ${ }^{1}$ P. Chenevier, ${ }^{1}$ M. Pinault, ${ }^{2}$ H.-J. Jeong, ${ }^{3}$ S. Enouz-Vedrenne, ${ }^{3}$ C. S. Cojocaru, ${ }^{3}$ D.

\section{ABSTRACT}

A versatile chemical vapor deposition (CVD) based method for the fabrication and electrical measurement of individual carbon nanotube junctions was developed. ferritin or Fe particles were grafted on multiwalled carbon nanotubes (MWNTs) and used as catalysts for the subsequent growth of secondary MWNT by CVD. Junctions were then individually connected. The conductivities of the MWNTs and of the junction were measured. Statistical data show that the conductance of the MWNTMWNT junction is similar to that of MWNT. This result paves the way for the use of carbon nanotubes as electrical interconnects in electronic applications.

Carbon nanotubes (CNTs) can be used in future electronics both as interconnects ${ }^{1,2}$ and as functional elements. ${ }^{3}$ In particular, CNTs have demonstrated higher electrical conductivity and current density ${ }^{4,5}$ than $\mathrm{Cu}$ and could avoid difficulties foreseen in nanometer scale $\mathrm{Cu}$ interconnects due to electromigration and mechanical instability. Therefore CNTs are appealing candidates for wiring in electronics circuits. ${ }^{6}$ Several examples of CNT vias ${ }^{7,8}$ and interconnects ${ }^{9}$ have been reported recently. However, to develop CNT solutions for interconnects, highly conducting CNT-CNT junctions are required. As CNT to CNT cross contacts are poor, ${ }^{9}$ connections could be achieved by metal welding ${ }^{10}$ or direct CNT intermolecular $Y$ junction. ${ }^{11,12}$ On the one hand, the electrical conductivity of CNT Y junctions has already been investigated, but only in the case of $\mathrm{Y}$ junctions obtained in a single growth step. ${ }^{13,14}$ However, building CNT interconnects requires the fabrication of T-type junctions by a multiple step process. On the other hand, regrowth of CNT on CNT has been demonstrated ${ }^{15-17}$ but the conductivity of those T-type junctions has not been measured and neither was their integration into CNT-wiring networks explored.

In the present paper, we describe the fabrication and electrical characterization of individual CNT junctions using chemical vapor deposition (CVD) regrowth. The selective placement of catalyst particles was achieved either by chemical coupling or by electrodirected placement. In a first approach, multiwalled CNTs (MWNTs) were functionalized either covalently with ferritin protein ${ }^{18}$ or noncovalently ${ }^{17,19}$ by hydrophobic iron oxide nanoparticles $\left(\mathrm{FeO}_{x} \mathrm{NPs}\right)$. Alternatively, bare MWNTs were first electrically connected and $\mathrm{FeO}_{x} \mathrm{NPs}$ were deposited thereon using dielectrophoresis (DEP). In all cases, MWNT-catalyst conjugates were deposited on a $\mathrm{SiO}_{2}$ substrate and subsequently calcinated and reduced, yielding MWNT supported $\mathrm{Fe}(0)$ particles used as catalyst for the CVD growth of new MWNTs.
In order to realize and electrically connect individual CNT junctions, sample preparation had to be carefully optimized. Namely single regrown CNTs on well separated MWNTs were preferred. First, MWNT-catalyst conjugates were spread at low density on a substrate patterned with an alignment grid so that individual, well separated MWNTs could be localized. Second, the catalyst nature and grafting yield as well as the CVD-growth parameters were fine tuned to obtain suboptimal regrowth yield, and thus favor single regrowth events. MWNT-catalyst conjugates (primary MWNTs) were localized and imaged before and after CVD growth in order to identify newly grown (secondary) MWNTs without ambiguity. Isolated CNT junctions were electrically connected using conventional e-beam lithography and the electrical characteristics of the MWNTs and the junction were measured.

Ferritin was coupled to MWNT through a well-known amide-bond strategy. For this purpose, carboxyl moieties were generated by acid treatment ${ }^{20}$ onto the aerosol-assisted CVD produced MWNTs. ${ }^{21}$ MWNTs were then incubated with carbodiimide and $N$-hydroxysuccinimide in aqueous buffer and reacted in situ with ferritin ${ }^{20}$ in a ferritin/MWNT mass ratio of 3:2. The obtained MWNT-ferritin conjugates showed a quite high coverage with an average density of about 10 ferritin molecules per $100 \mathrm{~nm}$ of MWNT length as evidenced by transmission electron microscopy (TEM) [Fig. 1(a)].

$\mathrm{FeO}_{x} \mathrm{NPs}$ were obtained by precipitation of iron oleate in boiling dioctylether according to Park et $a .^{22}$ Due to the hydrophobicity of their oleate shell, $\mathrm{FeO}_{x} \mathrm{NPs}$ have a good

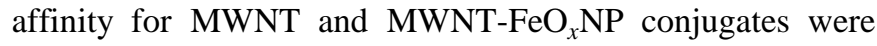
obtained by simply coincubating MWNT with $\mathrm{FeO}_{x} \mathrm{NP}$ (Ref. 20) (mass ratio 1:60). TEM images of the conjugates [Fig. 1(b)] showed MWNT covered with $5 \mathrm{~nm}$ particles. The average coverage was about $40 \mathrm{FeO}_{x} \mathrm{NPs}$ per $100 \mathrm{~nm}$ of MWNT length, which is noticeably higher than for MWNTferritin conjugates. 


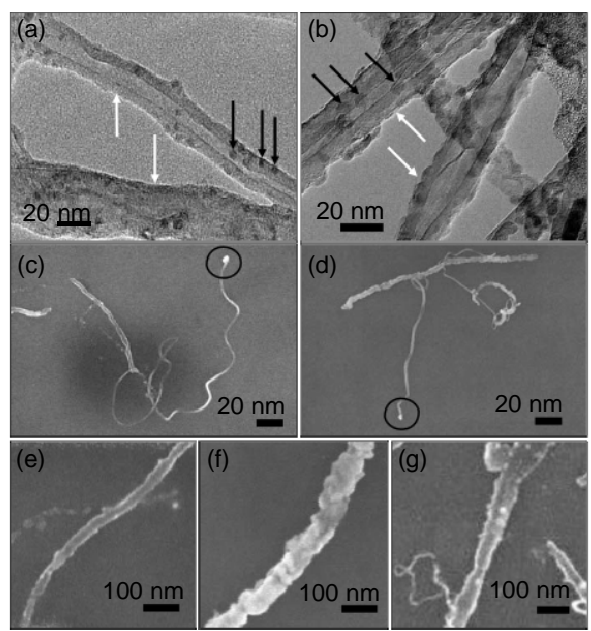

FIG. 1. TEM images of (a) MWNT-ferritin and (b) MWNT-FeO ${ }_{x}$ NP conjugates, showing CNTs (white arrows) decorated with metal particles (black arrows). SEM images of individual regrowth events from (c) MWNT-ferritin and (d) MWNT-FeO ${ }_{x} \mathrm{NP}$ conjugates. Circles show the probable location of the catalyst particle. SEM images showing the primary MWNTs after CVD

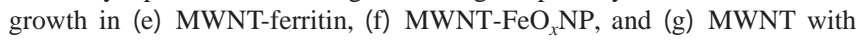
DEP deposited $\mathrm{FeO}_{x} \mathrm{NP}$ samples.

Prior to CVD growth, the samples were calcinated in air at $550{ }^{\circ} \mathrm{C}$ for $10 \mathrm{~min}$ to remove the organic shell. CNT growth was performed in an acetylene CVD reactor as described earlier. ${ }^{23}$ The reduction $\left(\mathrm{H}_{2}, 20 \mathrm{~min}\right)$ of the iron ox ide catalysts into $\mathrm{Fe}(0)$ was followed by CVD growth $\left(\mathrm{C}_{2} \mathrm{H}_{2}\right.$, 10 min). ${ }^{20}$ We first checked that ferritin and $\mathrm{FeO}_{x} \mathrm{NP}$ induced CNT growth in our CVD process and optimized the process for growth of small MWNTs on $\mathrm{SiO}_{2}$ substrates. ${ }^{20}$ MWNT growth was preferred to single walled nanotube (SWNT) (Ref. 24) growth from ferritin because individual CNT to CNT junction selection and electrical connection required scanning electron microscopy (SEM) imaging, the resolution of which does not allow individual SWNT detection. The CVD temperature was thus set at $700{ }^{\circ} \mathrm{C}$ to produce small MWNTs. ${ }^{23}$ In this process, ferritin and $\mathrm{FeO}_{x} \mathrm{NP}$ deposited on a $\mathrm{SiO}_{2}$ substrate yielded MWNT of similar diameters (5-20 $\mathrm{nm})$. In CVD regrowth from the MWNT-catalyst conjugates, the catalyst particles often appeared at the tip of the secondary MWNT [Figs. 1(c) and 1(d)], which suggests a tipgrowth mechanism.

SEM images of the MWNT-catalyst conjugates after CVD regrowth are shown in Figs. 1(e) and 1(f). MWNT-FeO ${ }_{x} \mathrm{NP}$ conjugates appear almost fully covered with packed particles, whereas MWNT-ferritin conjugates show sparse particles along the MWNT. In order to better control the $\mathrm{FeO}_{x} \mathrm{NP}$ coverage, DEP-based electrodeposition was performed. ${ }^{25}$ MWNTs were first deposited using DEP by applying an ac voltage between electrodes on a chip covered with a drop of MWNT solution. ${ }^{20}$ The same procedure was then used for the deposition of $\mathrm{FeO}_{x} \mathrm{NP}$ in toluene. The high aspect ratio of MWNTs increased DEP attraction toward their tip, ${ }^{26}$ favoring $\mathrm{FeO}_{x} \mathrm{NP}$ deposition at the end of the contacted MWNT. Individual particles can be seen along the MWNT on Fig. 1(g).

Examples of individual CNT junctions are shown in Figs. 1(c) and 1(d). Secondary MWNTs were usually smaller and appeared brighter than primary MWNTs because they are protruding above the surface, ${ }^{20}$ therefore limiting electron transfer to the substrate. Unfortunately, their three-

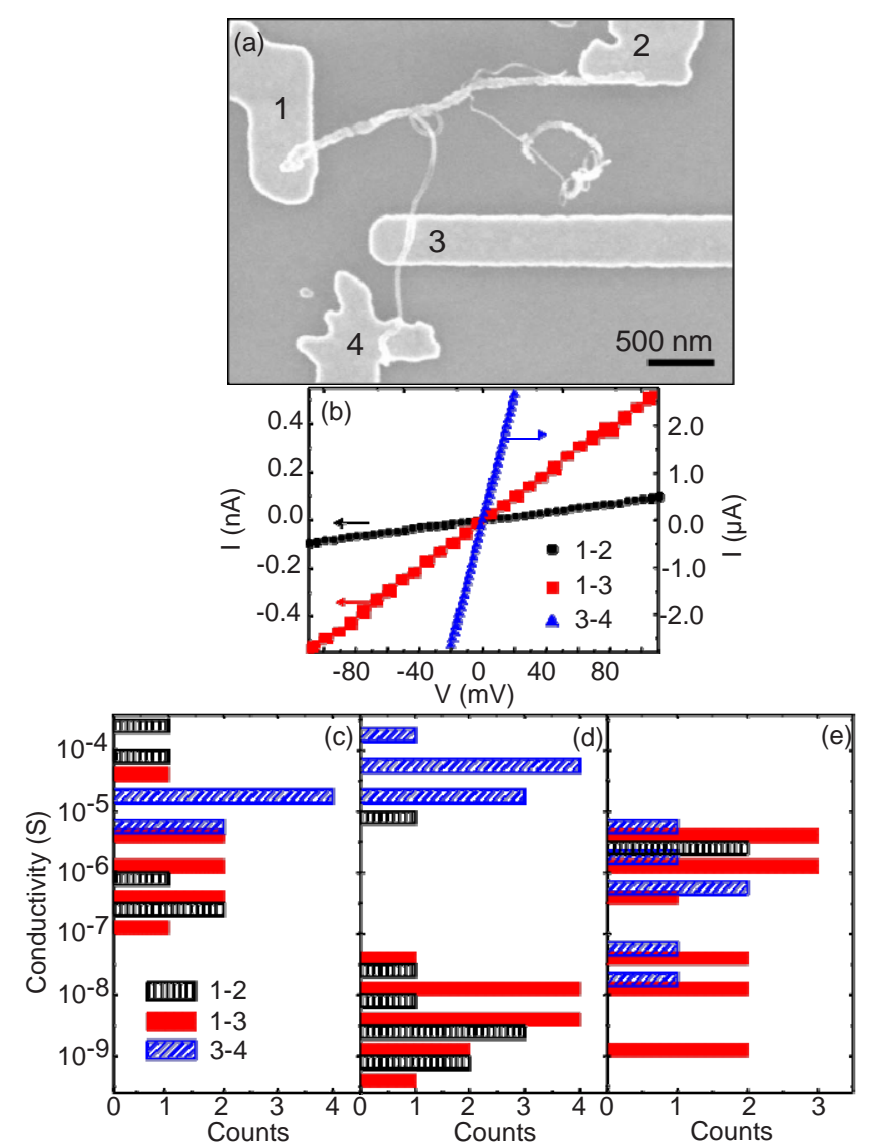

FIG. 2. (Color online) (a) SEM image and (b) $I-V$ characteristics of an

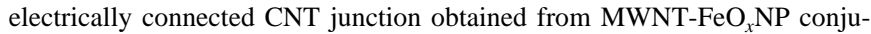
gates. [(c)-(e)] Histograms of conductances (see Ref. 20) for (c) MWNTferritin, (d) MWNT-FeO ${ }_{x}$ NP conjugate, and (e) DEP deposited MWNT and $\mathrm{FeO}_{x} \mathrm{NP}$ samples. The primary CNT is measured between electrodes 1 and 2, the secondary CNT between 3 and 4 and the junction between 2 and 3 .

dimensional shape prevented metal connection by e-beam lithography. Therefore, we used a wetting/dewetting cycle in acetone to bend the protruding MWNTs under the meniscus pressure and have them stick to the substrate. After process optimization, approximately 100 CNT junctions were detected on a grid, from which approximately 20 were connected through e-beam lithography and metallization (Pd 5 $\mathrm{nm} / \mathrm{Au} 50 \mathrm{~nm})$.

A typical example of an electrically connected CNT junction structure and its current-voltage $(I-V)$ characteristics are shown in Figs. 2(a) and 2(b). Statistics of the MWNT and junction conductances are shown in Figs. 2(c)-2(e). In all samples, secondary MWNTs were highly conducting (a few microamperes under $100 \mathrm{mV}$ dc bias) and always showed a linear $I-V$ curve. On the contrary, the primary MWNT conductance varied strongly according to the catalyst [Figs. 2(d) and 2(e)]. Their $I-V$ curves were in most cases linear and symmetric, except for a slight S-shape in some highly resistive cases. All samples were fabricated using the same primary MWNTs and thus should show similar intrinsic conductances. The catalyst coupling method should not affect their conductance either since the organic shell around the catalyst was burnt out during calcination. Therefore, the strong conductivity drop in the case of $\mathrm{MWNT}-\mathrm{FeO}_{x} \mathrm{NP}$ was most likely due to the dense $\mathrm{FeO}_{x} \mathrm{NP}$ coverage around the primary MWNTs, as can be seen in Fig. 1(f), which probably prevented direct electrical connection to the electrode. This 
drawback could nevertheless be overcome by the more controlled DEP deposition of $\mathrm{FeO}_{x} \mathrm{NP}$ [Fig. 2(g)]. As a first estimate, although the contact resistance with the metal electrode could rise up as high as gigaohms, the average conductance of the primary and secondary MWNTs lies probably in the $1-100$ f.LS range, in good agreement with previous reports. ${ }^{7,9}$

In the case of the CNT junction, the measured conductance was limited by the bottleneck of the conductance path between the $\mathrm{Au}$ electrodes, which included two metalMWNT contacts, two MWNT sections, and the CNT junction itself. The measured conductance of the CNT junction was usually intermediate between the conductance recorded for the primary and secondary MWNTs, which indicates that the CNT junction itself was not the conductance bottleneck. Our CNT junctions could sustain as high as 2 f.LA current.

The effect of gating on the conductance was also studied using a silicon backgate. ${ }^{20}$ A very small or no change was observed in the conductance of CNT junctions and primary MWNTs. In the case of secondary MWNTs, a negative gate voltage produced a small $(<50 \%)$ current increase. This is most likely due to some of the outer shells being semiconducting, as has been described for MWNTs of similar diameter. $^{27}$

As a conclusion, the CNT junctions appeared to behave as low resistivity contact elements, as needed for CNT-CNT interconnect use in future electronics. Although the molecular structure of our CNT junctions could not be investigated in detail by TEM, high resolution SEM observation of our regrown sample indicates a probable tip growth process, which implies that the catalyst particle moves away from the junction during the growth. A direct carbon on carbon connection is thus to be expected, as had been described in previous work. ${ }^{12,16}$

Applying a versatile CVD-regrowth technique, we fabricated for the first time individual MWNT-MWNT junctions and characterized them electrically. Our method can be tuned to a large extent since catalysts could be bound to the initial MWNT by three independent methods, covalent bonding, hydrophobic association, or DEP driven electrodeposition, all affording similar CVD regrowth yields. Although some of our measurements were hindered by high contact resistance, both primary and secondary MWNTs showed conductivities in the 1-100 f.LS range. The CNT junction itself did not noticeably decrease the conductivity of the MWNT-network, the metal to primary MWNT contact or the MWNTs themselves being the conductance bottleneck. Our work thus shows that highly conducting CNT interconnects can be developed from CVD growth technique.

The authors want to thank V. Derycke for his continuous interest and support in this work. We acknowledge the financial support from French ANR (Nanoreseaux project) and from Region Ile de France for funding our nanofabrication facility (SESAME project).

${ }^{1}$ A. P. Graham, G. S. Duesberg, R. Seidel, M. Liebau, E. Unger, F. Kreupl, and W. Höenlein, Diamond Relat. Mater. 13, 1296 (2004).

${ }^{2}$ J. Robertson, Mater. Today 10, 36 (2007).

${ }^{3}$ P. Avouris and J. Chen, Mater. Today 9, 46 (2006).

${ }^{4}$ A. Bachtold, M. Henny, C. Terrier, C. Strunk, C. Schonenberger, J. P. Salvetat, J. M. Bonard, and L. Forro, Appl. Phys. Lett. 73, 274 (1998).

${ }^{5}$ P. Poncharal, C. Berger, Y. Yi, Z. L. Wang, and W. A. de Heer, J. Phys. Chem. B 106, 12104 (2002).

${ }^{6}$ H. Li, W. Y. Yin, K. Banerjee, and J. F. Mao, IEEE Trans. Electron Devices 55, 1328 (2008).

${ }^{7}$ J. Li, Q. Ye, A. Cassell, H. T. Ng, R. Stevens, J. Han, and M. Meyyappan, Appl. Phys. Lett. 82, 2491 (2003).

${ }^{8}$ Y. Awano, S. Sato, D. Kondo, M. Ohfuti, A. Kawabata, M. Nihei, and N. Yokoyama, Phys. Status Solidi A 203, 3611 (2006).

${ }^{9}$ G. F. Close, S. Yasuda, B. C. Paul, S. Fujita, and H. S. P. Wong, IEEE Trans. Electron Devices 56, 43 (2009).

${ }^{10}$ Y. Peng, T. Cullis, and B. Inkson, Nano Lett. 9, 91 (2009).

${ }^{11}$ D. Wei and Y. Liu, Adv. Mater. 20, 2815 (2008).

${ }^{12}$ P. R. Bandaru, J. Mater. Sci. 42, 1809 (2007).

${ }^{13}$ D.-H. Kim, J. Juang, H. K. Shin, S. Roy, and W. Choi, Nano Lett. 6, 2821 (2006).

${ }^{14}$ P. R. Bandaru, C. Daraio, S. Jin, and A. M. Rao, Nature Mater. 4, 663 (2005).

${ }^{15}$ N. Li, X. Chen, L. Stoica, W. Xia, J. Qian, J. Aßmann, W. Schuhmann, and M. Muhler, Adv. Mater. 19, 2957 (2007).

${ }^{16}$ X. Lepro, Y. Vega-Cantu, F. J. Rodriguez-Macias, Y. Bando, D. Golberg, and M. Terrones, Nano Lett. 7, 2220 (2007).

${ }^{17}$ E. Unger, G. S. Duesberg, M. Liebau, A. P. Graham, R. Seidel, F. Kreupl, and W. Höenlein, Appl. Phys. A: Mater. Sci. Process. 77, 735 (2003).

${ }^{18}$ S. Bhattacharyya, C. Sinturel, J. P. Salvetat, and M. L. Saboungi, Appl. Phys. Lett. 86, 113104 (2005).

${ }^{19}$ J. Li, S. Tang, L. Lu, and H. C. Zeng, J. Am. Chem. Soc. 129, 9401 (2007).

${ }^{20}$ See EPAPS supplementary material at http://dx.doi.org/10.1063/ 1.3216839 for more experimental details.

${ }^{21}$ M. Pinault, V. Pichot, H. Khodja, P. Launois, C. Reynaud, and M. MayneL'Hermite, Nano Lett. 5, 2394 (2005).

${ }^{22}$ J. Park, K. An, Y. Hwang, J.-G. Park, H.-J. Noh, J.-Y. Kim, J.-H. Park, N.-M. Hwang, and T. Hyeon, Nature Mater. 3, 891 (2004).

${ }^{23}$ H.-J. Jeong, L. Eude, M. Gowtham, B. Marquardt, S. H. Lim, S. Enouz, C. S. Cojocaru, K. A. Park, Y. H. Lee, and D. Pribat, NANO 3, 145 (2008).

${ }^{24}$ Y. Li, W. Kim, Y. Zhang, M. Rolandi, D. Wang, and H. Dai, J. Phys. Chem. B 105, 11424 (2001).

${ }^{25}$ L. Zheng, S. Li, J. P. Brody, and P. J. Burke, Langmuir 20, 8612 (2004).

${ }^{26}$ S. Tuukkanen, J. J. Toppari, A. Kuzyk, L. Hirviniemi, V. P. Hytönen, T. Ihalainen, and P. Törmä, Nano Lett. 6, 1339 (2006).

${ }^{27}$ P. C. Collins, M. S. Arnold, and P. Avouris, Science 292, 706 (2001). 